\title{
STATUT PORAL DES SOLS CULTIVES DANS LA ZONE COTONNIERE OUEST DU BURKINA FASO
}

\author{
B. OUATTARA ${ }^{1}$, K. OUATTARA ${ }^{1}$, M. P. SEDOGO $^{1}$ et A. YAO-KOUAME ${ }^{2}$ \\ ${ }^{1}$ Institut de l'Environnement et de Recherches Agricoles, INERA, 04 BP 8645 Ouagadougou 04. \\ E-mail : badiori.ouattara@coraf.org. \\ 2UFR/STRM, Université de Cocody-Abidjan, 22 BP 582 Abidjan 22
}

\begin{abstract}
RESUME
L'amélioration de l'état physique du sol est essentielle pour une productivité agricole soutenue. Une étude diagnostic du statut poral a été conduite sur des Lixisols et Luvisols ferriques du Burkina Faso, en vue d'évaluer les effets cumulatifs des systèmes de culture. Cent-une parcelles ont été échantillonnées, à partir d'une typologie des grands systèmes de culture (itinérante, cyclique, permanente) et de la fréquence des labours (occasionnel, bisannuel, annuel). La porosité du sol a été mesurée au densitomètre à membrane, in situ, et au porosimètre à mercure, sur l'horizon $0-15 \mathrm{~cm}$. Les résultats montrent que la mise en culture permet de porter le statut poral des sols sous jachère, de 41 à $45 \%$. Mais, ces modifications portent, notamment, sur la porosité structurale (macroporosité) du sol. Les labours annuels induisent, à terme, une perte de macroporosité de près de deux points, comparativement à la situation sous culture itinérante. Ils contribuent à fusionner les familles de macropores $(50-100 \mu \mathrm{m})$ et de micropores $(10 \mu \mathrm{m})$ des sols sous jachère naturelle, pour créer une nouvelle famille de pores de $15-25 \mu \mathrm{m}$ de diamètre équivalent.
\end{abstract}

Mots-clés : Lixisol, Luvisol, systèmes de culture, porosité du sol.

\section{ABSTRACT}

CULTIVATION-INDUCED EFFECTS ON SOIL POROSITY IN THE WESTERN COTTON AREA OF BURKINA FASO

The improvement of soil physical properties is of paramount importance in ensuring sustainable crop production. A diagnostic study on soil porosity was carried out on a Ferric Lixisol and a Ferric Luvisol from Burkina Faso, in order to assess the cumulative effects of cropping systems. One hundred-and-one plots were sampled based on the typology of major cropping systems (shifting, cyclic, continuous cultivation) and tillage intensity (occasional, biennial, annual ploughing). Soil porosity was measured within the top soil layer (0$15 \mathrm{~cm}$ ), with a rubber-balloon densitometer, in situ, and with a mercury porosimeter. The results showed that soil cultivation increased the porosity of soils under cyclic fallow, from 41 to $45 \%$. But, these modifications affected mainly soil structural porosity (macroporosity). Annual ploughings induced, in the long-term, a loss, by two points, of soil structural porosity, as compared to shifting cultivation fallow systems. They contributed to combine soil macropore groups $(50-100 \mu \mathrm{m})$ and micropores $(10 \mu \mathrm{m})$ under natural fallow into a new pores group of equivalent diameters $(15-25 \mu \mathrm{m})$.

Key words : Lixisol, Luvisol, cropping systems, soil porosity.

\section{INTRODUCTION}

Le maintien d'un bon statut poral des sols cultivés est un enjeu majeur pour les agriculteurs, dans l'optique d'une gestion durable de la fertilité des terres agricoles. Cette caractéristique physique du sol conditionne, pour une large part, son aptitude à mettre à la disposition des plantes, via un bon développement du système racinaire, l'eau, l'oxygène et les éléments nutritifs nécessaires à leur croissance (Hénin et al., 1969 ; Boiffin et Marin-Laflèche, 1990). Aussi, les paysans des savanes soudaniennes, ont-ils, pendant longtemps, utilisé la pratique de la jachère pour maintenir la capacité productive de leurs sols, après des phases culturales plus ou moins 
longues (Ruthenberg, 1971; Nye et Greenland, 1965 ; Serpantié et Ouattara, 2001). Mais de nos jours, du fait des besoins d'intensification agricole et des conséquences de la pression démographique, la pratique de la jachère fait de plus en plus place à la culture permanente, avec des impacts plus ou moins dommageables sur la fertilité des sols (Ouattara et al., 1994 ; 1998 ; 2006).

La caractérisation du système poral des sols cultivés a, certes, fait l'objet de nombreuses études (Charreau et Nicou, 1971 ; Sarr, 1981; Boiffin et Marin-Laflèche, 1990 ; Nicou et al., 1990 ; Ouattara et al., 1994 ; 1998). Mais, force est de constater qu'au regard de la grande hétérogénéité qui affecte, d'une façon générale, l'état physique des horizons de surface des sols cultivés, ces études ont surtout porté sur des dispositifs expérimentaux en milieu contrôlé. Très peu de ces travaux ont été menés en milieu paysan où les systèmes de culture, très souvent contrastés, se côtoient dans un petit espace agricole, comme c'est le cas dans la zone cotonnière Ouest du Burkina Faso.

La présente étude vise à analyser, l'impact de ces systèmes de culture sur l'évolution du statut poral, et à juger de leur efficacité en terme de gestion durable de la fertilité des sols.

\section{MATERIEL ET METHODES}

\section{SITE D'ETUDE}

L'étude a été menée à Bondoukui, $11^{\circ} 51^{\prime} \mathrm{N}$, $3^{\circ} 46^{\prime} \mathrm{W}, 360 \mathrm{~m}$, dans la zone cotonnière Ouest du Burkina Faso. La pluviométrie annuelle moyenne se situe entre 900 et $1000 \mathrm{~mm}$ et est distribuée de façon monomodale entre Mai et Octobre. Les températures maxima se situent autour de $30^{\circ} \mathrm{C}$. L'évapotranspiration potentielle atteint en moyenne $1900 \mathrm{~mm}$. Selon la classification FAO (1998), les sols appartiennent à l'ordre des Lixisols ferriques (texture sablolimoneuse) et des Luvisols ferriques (texture limono argileuse à argileuse, $>15 \%$ d'argile). Leurs principales caractéristiques physicochimiques sont mentionnées dans le tableau 1. Les principaux types de formations végétales rencontrées dans la région de Bondoukui correspondent à la végétation liée au réseau hydrographique (forêt galerie) et aux formations savanicoles (savane arbustive, savane arborée ou savane herbeuse, forêt claire). Toutefois, les espaces cultivés sont dominés par d'importants parcs à karité (Vitellaria paradoxa) et à néré (Parkia biglobosa) (Devineau et al., 1997).

Tableau 1 : Caractéristiques physico-chimiques des sols sous jachère naturelle.

Soil chemical and physical characteristics under natural fallow.

\begin{tabular}{ll}
\hline Granulométrie (\%.) & \\
Sables & 56 \\
Limons & 31 \\
Argiles & 13 \\
\hline Matière organique $\left(\mathrm{T} . h a^{-1}\right)$ & \\
Carbone & 11,8 \\
Azote & 0,9 \\
\hline $\mathrm{Complexe}^{2+}$ absorbant (Cmol(+)/kg-Sol) & \\
$\mathrm{Ca}^{2+}$ & 2,22 \\
$\mathrm{Mg}^{2+}$ & 0,82 \\
$\mathrm{Na}^{+}$ & 0,04 \\
$\mathrm{~K}+$ & 0,19 \\
Somme des bases (S) & 3,1 \\
Capacité d'échange (T) & 3,6 \\
Saturation (\%) & 86 \\
\hline pHeau & 6,1 \\
\hline
\end{tabular}




\section{CHOIX DES PARCELLES D'ETUDE}

Les parcelles ont été échantillonnées à partir d'une typologie des exploitations agricoles en fonction de leur mode de mise en valeur. Trois grands systèmes de culture (GSC) ont été ainsi identifiés :

- le système de culture itinérante, caractérisé par 5 à 10 ans de culture pour plus de 30 ans de jachère ; ces vieilles jachères sont localement appelées diuré ;

- le système de culture à jachère ou de culture cyclique, caractérisé par 5 à 10 ans de culture suivi de 5 à 20 ans de jachère ;

- le système de culture permanente, avec plus de 20 ans de cultures quasiment ininterrompues.
Ces GSC ont été recoupés avec l'âge des phases culture- jachère et le régime du travail du sol (Tableau 2). Des champs qui reçoivent des apports de matière organique sous forme de déjections animales ou de compost ont également été identifiés, dans les systèmes de culture permanente. Les doses apportées varient entre 2 et 5 t.ha $^{-1}$, à des fréquences également variables (2 à 3 ans).

Les labours ont été réalisés, en attelé ou en motorisé, à des profondeurs d'au plus $15 \mathrm{~cm}$. L'échantillonnage du sol a été effectué à l'échelle de la parcelle de culture. Au total, 101 parcelles, dont 33 sous jachères naturelles, ont été échantillonnées pendant la saison sèche. Les prélèvements ont été réalisés consécutivement aux mesures de densité apparente sèche du sol, sur une profondeur de $15 \mathrm{~cm}$, et à raison de trois répétitions par parcelle.

Tableau 2 : Répartition des parcelles entre les différents systèmes de culture.

Plots distribution between the different cropping systems.

\begin{tabular}{|c|c|c|c|c|c|c|c|c|}
\hline & \multicolumn{2}{|c|}{ Système Itinérant } & \multicolumn{3}{|c|}{ Systèmes à jachère } & \multicolumn{3}{|c|}{ Système permanent } \\
\hline & $\mathrm{J} 30$ & $\mathrm{C} 10$ & $\mathrm{~J} 10$ & $\mathrm{~J} 20$ & $\mathrm{C} 10$ & $\mathrm{Lab} / 2 \mathrm{an}$ & Lab./an & Fumier \\
\hline N. parcelles & 11 & 7 & 9 & 13 & 23 & 16 & 15 & 6 \\
\hline
\end{tabular}

\section{CARACTERISATION DU SYSTEME PORALDU SOL}

La mesure du système poral du sol a été effectuée par densitométrie, à partir des mesures de la densité apparente sèche, Da, réalisée, in situ, à l'aide du densitomètre à membrane sur l'horizon 0 - $15 \mathrm{~cm}$ (Stengel, 1979).

La porosité totale, $\mathrm{n}(\mathrm{pc})$, du sol a été déterminée à l'aide de la formule suivante :

$$
n=100\left(1-\frac{D a}{D r}\right)
$$

où Dr est la densité réelle du sol, déterminée par picnométrie à eau au laboratoire (Blake, 1965).

La composante texturale de la porosité du sol, résultant de l'assemblage intime de ses constituants, a été obtenue à l'aide de la mesure des densités texturales $(\mathrm{Dt})$, réalisée au laboratoire, sur des "gâteaux texturaux" (Stengel, 1979 ; Ouattara et al., 1998). La porosité du «gâteau textural» $(\mathrm{nT})$, est obtenue à l'aide de la formule suivante :

$$
n T=100\left(1-\frac{D t}{D r}\right)
$$

Rapportée à l'échantillon naturel de sol, la porosité texturale, nt, devient :

$$
n t=100 D a\left(\frac{1}{D t}-\frac{1}{D r}\right)
$$

La porosité structurale (ns), issue de l'action combinée des facteurs externes (climatique, mécanique et biologique), est obtenue par la différence entre la porosité totale et la porosité

$$
\begin{aligned}
& \text { texturale du sol : } \\
& n s=n-n t=100\left(1-\frac{D a}{D t}\right)
\end{aligned}
$$




\section{DETERMINATION DU SPECTRE PORAL DU SOL}

L'injection de mercure a été utilisée pour décrire la distribution de la taille des pores du sol. Le principe repose sur le fait qu'il faut exercer une pression d'autant plus forte, pour faire pénétrer le mercure dans un matériau poreux, que la taille des pores est petite. La loi de Jurin associe les volumes de mercure intrudé à différentes pressions $(P)$ aux diamètres équivalents des pores (d) supposés de forme simple et régulière, selon l'équation (Jurin, 1718) :

$$
P=\frac{2 \gamma \operatorname{Cos} \alpha}{d}
$$

où $\gamma$ est la tension superficielle du mercure et $\alpha$, l'angle de raccordement du ménisque à l'interface mercure-solide. Les valeurs de $\gamma$ et de $\alpha$ utilisées ont été, respectivement, de 484 dynes $/ \mathrm{cm}$ et de $130^{\circ}$ (Pellerin, 1980).

L'appareil, utilisé pour la mesure du volume des pores de diamètre compris entre 0,005 et $300 \mu \mathrm{m}$, est un porosimètre Micromeritric 9310 . Les mesures ont été réalisées sur des mottes de terre, de 5 à $8 \mathrm{~cm}^{3}$ de volume, préalablement séchées à l'étuve et, à raison de 2 répétitions par échantillon.

\section{TRAITEMENT STATISTIQUE DES DONNEES}

Les analyses de variance ont été réalisées à l'aide du logiciel Genstat 6 ® version 3.2 (2000). Pour ce faire, l'ensemble des variables descriptives des GSC a été codé de 1 à 8 .

\section{RESULTATS}

\section{LES SYSTEMES DE POROSITE DU SOL}

L'expression des porosités texturale et structurale du sol en fonction de la porosité totale, permet de suivre l'évolution induite de ces deux paramètres avec les pratiques culturales (Figure 1). La porosité texturale est demeurée pratiquement constante, quelles que soient les valeurs de la porosité totale du sol. Toutefois, une certaine variabilité a affecté le diagramme de dispersion de la fraction texturale de la porosité du sol (Figure 2 et Tableau 3 ). La porosité structurale (ns), quant à elle, a augmenté en fonction des valeurs croissantes de la porosité totale $(\mathrm{n})$ du sol : $\mathrm{ns}=1,2 \mathrm{n}-32,5$, avec $\mathrm{R}^{2}=$ 0,78 ( $R^{2}$ est le coefficient de corrélation).
Les jachères du système de culture itinérante ont pu maintenir un bon statut poral des sols $(44,5 \%)$, tandis que les sols des jachères du système cyclique sont apparus particulièrement plus tassés, avec une porosité totale de $41 \%$ (Tableau 3). Ce volume poral monte à $44 \%$ lorsqu'ils ont été mis en culture. Toutefois, les parcelles annuellement labourées ont induit, à moins d'une quinzaine d'années de culture, des baisses de près de 2 points de porosité structurale par rapport à la situation sous les vieilles jachères du système de culture itinérante (Tableau 3).

\section{ANALYSE DU SPECTRE DE POROSITE}

Les résultats obtenus avec les essais d'imprégnation au mercure ont été exprimés sous forme de courbe de distribution des volumes poraux en fonction des diamètres d'accès des pores. Ils permettent d'analyser, de façon qualitative, non seulement l'effet des variations granulométriques des sols, mais aussi les changements apparus au niveau de la taille et de la répartition des réseaux de pores, suite aux labours des sols précédemment sous jachères naturelles (Figures 3 et 4). L'observation des spectres de porosité des sols montre que la distribution du volume poral est liée aux variations de la granulométrie du squelette sableux, associé à la phase argileuse. Ceci a été traduit à l'aide d'un indice granulométrique (lg) du sol, comme suit :

$$
I g=\frac{A}{L G+S G}
$$

Où $A, L G$ et $S G$ représentent, respectivement, les teneurs en argiles, en limons grossiers et en sables grossiers. II apparaît que les spectres de porosité sont d'autant plus étalés que la granulométrie du sol est fine :

- dans les sols sableux, peu perturbés, sous jachère naturelle, ( $5 \%$ d'argile), pour lesquels l'indice granulométrique a évolué entre 0,1 et 0,20 , on observe un pic bien marqué, caractérisant une famille de pores largement dominants et centrés autour d'un diamètre de $50 \mu \mathrm{m}$ (Figures 3A, 3B) ;

- dans les sols plus argileux $(>15 \%)$ pauvres en sables grossiers $(0,4<\lg <0,8)$ la distribution des volumes des pores a présenté une allure quasiment tri modale. Ainsi, 3 familles nettement distinctes de pores s'étalent autour des diamètres équivalents de 0,$01 ; 0,5$ et $50 \mu \mathrm{m}$ (Figures 3C, 3D et 4); 
- l'effet du labour s'est traduit par la présence d'un spectre poral nettement bi modale et ce, quelle que soit la granulométrie des sols. Ainsi, le premier pic, généralement non modifié, correspond à la gamme de micro pores de 0,01 $\mu \mathrm{m}$ de diamètre. Le second pic caractérise la famille de pores plus gros, (diamètre $=16 \mu \mathrm{m}$ ) pour les sols à texture grossière et pauvres en argiles $(\mathrm{Ig}=0,1)$ et de $20 \mu \mathrm{m}$ pour les sols à texture plus équilibrée $(0,5<\lg <0,80)$.

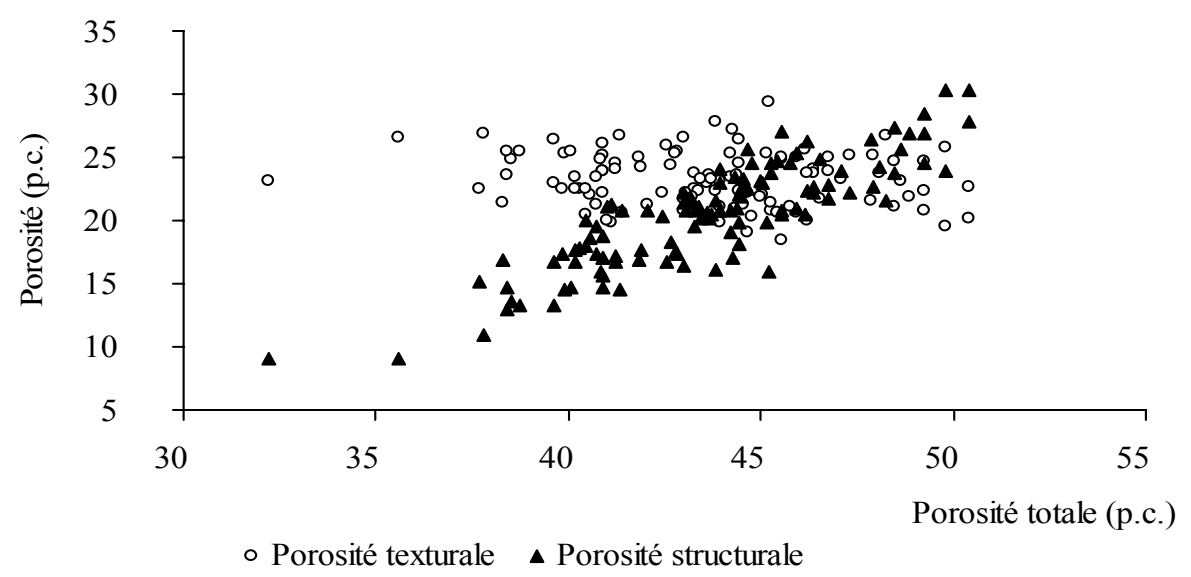

Figure 1 : Evolution des composantes texturale et structurale de la porosité des sols sous les effets induits des systèmes de culture.

Induced-effects of cropping systems on the textural and structural components of soil porosity.

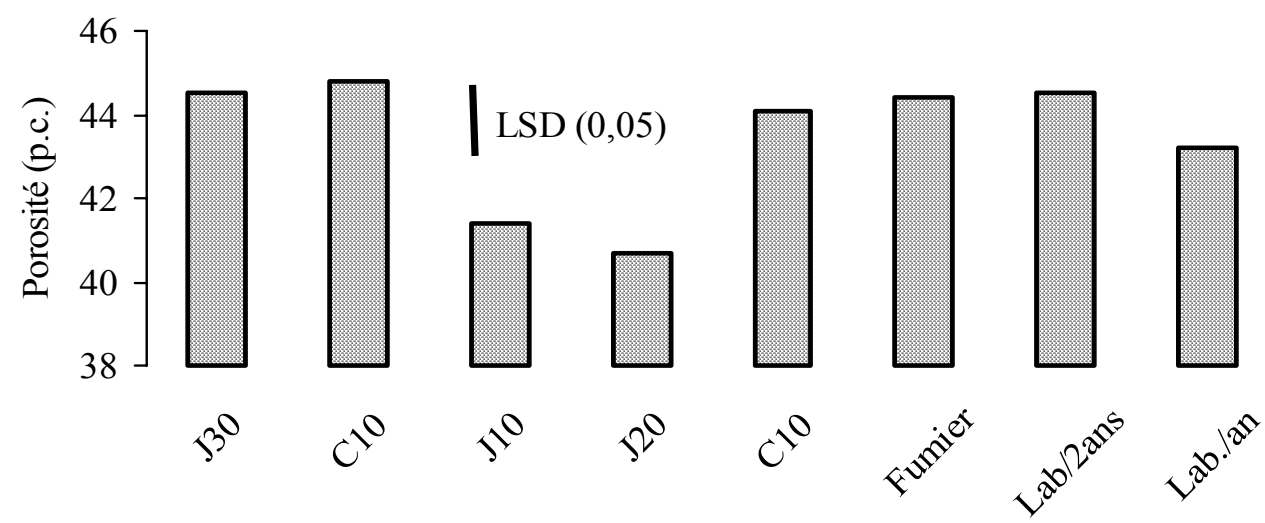

Système itinérant Système à jachère Système permanent

LSD : Least Significant Difference (Plus petite différence significative)

Figure 2 : Evolution de la porosité totale du sol en fonction des systèmes de culture.

Changes in soil porosity as function of cropping systems. 
Tableau 3 : Quantification du système poral du sol en fonction des systèmes de culture. Soil porosity determination as function of cropping systems.

\begin{tabular}{|c|c|c|c|c|c|c|c|c|c|c|}
\hline \multirow{2}{*}{ Porosité } & \multicolumn{2}{|c|}{ Syst. itinérant } & \multicolumn{3}{|c|}{ Système à Jachère } & \multicolumn{3}{|c|}{ Système permanent } & \multirow{2}{*}{ LSD } & \multirow{2}{*}{$\mathrm{P}<\mathrm{F}$} \\
\hline & $\mathrm{J} 30$ & $\mathrm{C} 10$ & $\mathrm{~J} 10$ & $\mathrm{~J} 20$ & $\mathrm{C} 10$ & $\mathrm{Lab} / 2 \mathrm{ans}$ & Fumier & $\mathrm{Lab} / \mathrm{an}$ & & \\
\hline $\mathrm{n}(\%)$ & 44,5 & 44,8 & 41,4 & 40,7 & 44,1 & 44,5 & 44,4 & 43,2 & 2,9 & 0,009 \\
\hline ns $(\%)$ & 21,8 & 20,9 & 17,9 & 17,1 & 21,1 & 21,2 & 21,5 & 20,2 & 1,9 & 0,05 \\
\hline nt $(\%)$ & 22,7 & 23,9 & 23,5 & 23,6 & 23,0 & 23,4 & 22,9 & 22,9 & - & NS \\
\hline
\end{tabular}

$\mathrm{J} 30$ (Jachère de 30 à 40 ans) ; J10 (Jachère de 1 à 10 ans) ; J20 (Jachère de 11 à 20 ans) ; C10 (Culture de 1 à 10 ans) ; Lab/2an (Labour bisannuel) ; Lab/an (labour annuel) ; N. parcelles (Nombre de parcelles).

$\mathrm{n}$ (porosité totale) ; ns (porosité structurale) ; nt (porosité texturale) ; NS (Non Significatif).
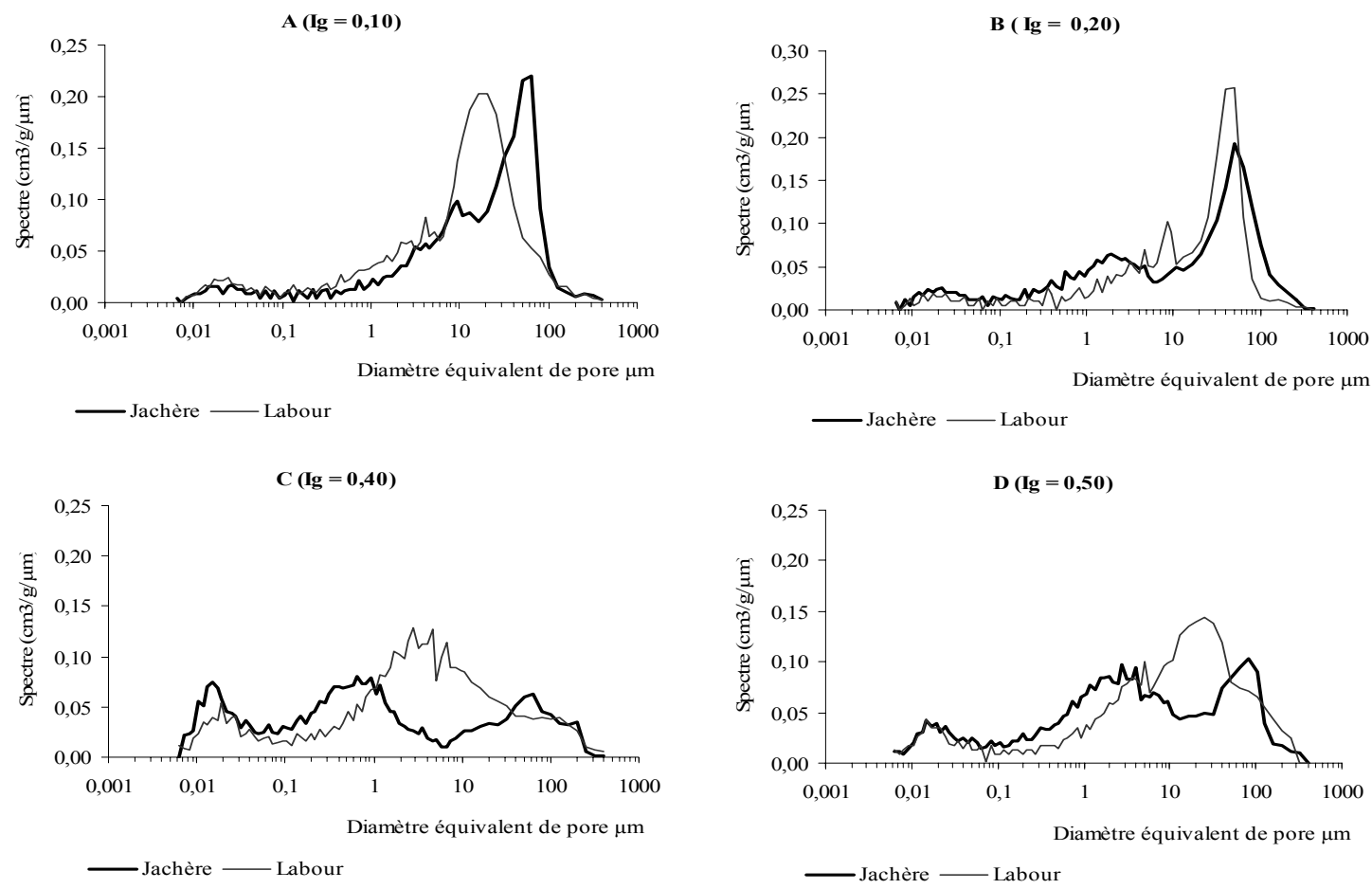

Figure 3 : Courbes de distribution des diamètres équivalents des pores dans les sols labourés et sous jachère naturelle à différentes gammes texturales.

Distribution curves of soil equivalent diameters pores in ploughed and fallow plots at different textural ranges.

Les lettres A, B, C, D identifient les différentes gammes texturales des sols, qui sont représentées par des indices de granulométrie (lg). The letters $A, B, C, D$ identify the various soil texture ranges, which are represented by texture ratings (lg). 


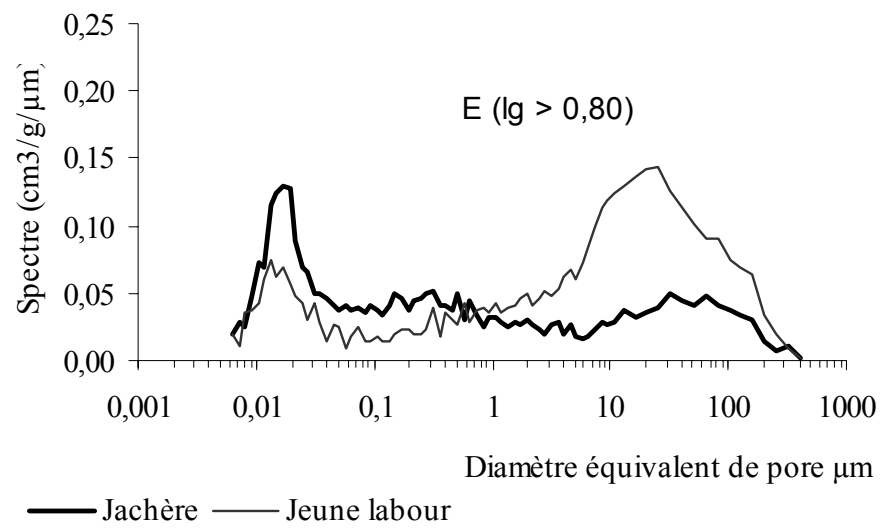

Figure 4 : Courbe de distribution des diamètres équivalents des pores dans des sols labourés et sous jachère.

Distribution curve of soil equivalent diameters pores in ploughed and fallow plots.

\section{DISCUSSION}

La caractérisation de l'espace poral des couches superficielles du sol est sujette à des difficultés liées, entre autre, à la grande complexité de l'organisation architecturale des sols, et surtout à la grande variabilité qui affecte le profil cultural (Stengel, 1979 ; Ouattara et al., 1998). Aussi, avons-nous opté pour l'utilisation de plusieurs outils méthodologiques pour mieux cerner les modifications induites par les différentes pratiques culturales sur la porosité des sols. Les avantages et les limites se rapportant à l'utilisation de ces différents outils sont également discutés.

Les valeurs de la porosité texturale ne sont pas significativement modifiées par les GSC bien qu'elles soient affectées par la variabilité liée à la large gamme texturale des sols du terroir de Bondoukui (Ouattara et al., 2006). C'est plutôt la porosité structurale ou macro porosité qui explique, à près de $80 \%$, les variations de l'espace poral des horizons superficiels des sols cultivés. En effet, les résultats de nos travaux antérieurs, obtenus en station de recherche sur des sols à gamme texturale plus étroite, ont montré que la mise en culture des sols ne modifie pas significativement leur texture, mais plutôt leur structure (Ouattara et al., 1998). Ils sont également en adéquation avec ceux de Kherabi et Monnier (1968); Stengel (1979) sous climat tempéré.

Le diagnostic des effets induits des GSC sur le statut poral des sols montre que les sols sous jachères naturelles apparaissent plus tassés que ceux des champs cultivés. II s'agit notamment des jachères jeunes et des jachères d'âge intermédiaire (10 - 20 ans) qui, du fait de l'effet du piétinement par le bétail, affichent les plus faibles valeurs de porosité structurale. Tout se passe comme si, dès que la culture itinérante est abandonnée, les jachères, même de moyenne durée n'ont plus la capacité, du fait de leur histoire (sols particulièrement déstructurés et instables après une longue culture) de résister au piétinement du bétail. En effet le surpâturage est particulièrement plus intense dans les jeunes jachères que dans celles du système itinérant. Cette situation provient du fait que les jeunes jachères sont recolonisées par les herbacées annuelles plus appétées alors que les sous-bois des vieilles jachères sont plutôt constitués d'arbustes buissonnants, à très faibles valeurs fourragères.

Ce phénomène de compactage des sols, observé sous jachères naturelles, et mis en évidence aussi bien par densitométrie, in situ, que par porosimétrie au mercure, est d'autant plus accentué que la texture des sols est fine (Ouattara et al., 1998 ; Martinez et Zinck, 2004).

L'utilisation du porosimètre à mercure a permis, certes, de quantifier le système poral des sols. Mais, du fait de la taille très faible des mottes, elle ne permet de mesurer qu'une maille très restreinte du réseau poral du sol, comparativement aux mesures réalisées au densitomètre à membrane. Aussi, est-elle d'une application assez limitée dans l'analyse du 
statut poral des sols. Elle sera plutôt réservée à l'analyse, in fine, des modifications affectant la distribution des réseaux de pores et induites, aussi bien par la composition granulométrique du sol que par les pratiques culturales.

Le labour a contribué à améliorer surtout la macroporosité des sols. Aussi, cette pratique culturale a t-elle, pendant très longtemps, été recommandée pour une bonne préparation des lits de semences sur des sols ferrugineux de l'espace sahélien, se caractérisant par leur forte sensibilité à la prise en masse et à l'encroûtement (Nicou, 1974 ; Sarr, 1981). En revanche, la pratique continue des labours bisannuels à annuels, conduit, à la longue, à une destruction de la structure des sols (Ouattara et al., 1998). II en résulte, en moins d'une quinzaine d'années de culture, une baisse de près de deux points de la macro porosité des sols labourés par rapport à ceux sous diuré.

Toutefois, les apports de matière organique au sol (parcage des animaux ou apport de compost) ont pu maintenir un niveau de porosité proche de celui sous jachère naturelle du système de culture itinérante (diuré). Cette situation s'expliquerait par la présence des fractions libres de la matière organique, qui en s'intercalant entre les particules minérales du sol, augmentent la porosité globale du sol (Boiffin et al., 1990). Elle peut également s'expliquer par le rôle de protection des agrégats terreux de la fraction humifiée du sol qui crée une porosité de type porphyrique (Tisdall et Oades, 1982 ; Duval et al., 1993 ; Spaccini et al., 2001).

L'analyse du spectre de porosité des sols montre que les labours contribuent à fusionner la famille des gros pores $(50-100 \mu \mathrm{m}$ de diamètre) et celle des micropores $(10 \mu \mathrm{m})$. On aboutit alors à la création d'une nouvelle famille, couvrant une gamme plus élargie de gros pores dont les diamètres équivalents sont centrés autour de 16 et $20 \mu \mathrm{m}$, respectivement, dans les sols à texture grossière et dans les sols plus argileux. Cette observation est en accord avec les résultats de travaux de Vauclin et Chopart (1992), réalisés au Nord de la Côte d'Ivoire, qui ont montré que les labours contribuaient à créer un nombre plus élevé de pores fonctionnels de taille réduite, 20 - $30 \mu \mathrm{m}$ de diamètre. L'ensemble de ces modifications induites par le travail du sol dans la morphologie et la distribution du réseau des pores est également confirmé par de récents travaux (Hangen et al., 2002 ; Wahl et al., 2004 ; Coquet et al., 2005 ; Ouattara et al., 2006).

D'une façon générale, le spectre poral de toutes les parcelles labourées se caractérise par l'existence de deux familles de pores. La première famille, avec un diamètre équivalent de pores de 0,01 $\mu \mathrm{m}$, correspond à l'entrée du mercure dans la phase argileuse du sol (Fiès, 1984). Elle n'est pas modifiée par le travail du sol. C'est plutôt la seconde famille, beaucoup plus grande, qui résulte des effets induits du travail du sol tel que nous l'avions déjà montré.

\section{CONCLUSION}

L'analyse de la porosité des sols montre clairement que c'est véritablement au niveau des macro pores que s'opèrent les modifications majeures du système poral sous les effets induits des pratiques culturales. Ainsi, les labours contribuent, dans un premier temps, à améliorer la porosité des sols cultivés, comparativement à ceux sous jachères naturelles qui, du fait de la forte anthropisation, apparaissent plus tassés. Toutefois, ils détruisent les bio pores (galeries d'insectes) qui y sont plus abondants et qui assurent une bonne infiltration des eaux de pluie. Par ailleurs, lorsque les labours se répètent annuellement, comme c'est le cas dans les rotations coton-maïs, ils contribuent, à terme, à un effondrement de l'architecture des sols et à une baisse subséquente de leur porosité globale.

En somme, les jachères naturelles, telles que pratiquées dans le terroir de Bondoukui, ne parviennent plus à jouer leur fonction de restauration de la fertilité physique des sols d'antan. De nos jours, elles ont tendance à disparaître des paysages agricoles des zones cotonnières, du fait de l'intensification agricole. Aussi, en terme d'alternative, le maintien d'une couverture vivante du sol pourrait toujours être envisagé à travers la mise en place de jachères "artificialisées», à base de graminées ou de légumineuses (sole fourragère). Toutefois, cette pratique devra s'inscrire dans des systèmes de culture cohérents et viables, dans lesquels les techniques de semis directs seront privilégiées. 


\section{REFERENCES}

Blake G. R. 1965. Particle density. In : Black C.A. (Ed.), Methods of soils analysis - Part1, American Society of Agronomy. Madison, Wisconsin, pp 371 - 373.

Boiffin J. et A. Marin-Laflèche. 1990. La structure du sol et son évolution : conséquences agronomiques, maîtrise par l'agriculteur. Les colloques de l'INRA, $n^{\circ} 53$; Paris, Laon, France, $216 \mathrm{p}$.

Boiffin J., Guérif J. et P. Stengel. 1990. Les processus d'évolution de l'état structural du sol : quelques exemples d'études expérimentales récentes. In : Boiffin J. et $A$. Marin-Laflèche, (Eds.). La structure du sol et son évolution. Les colloques de I'INRA, $\mathrm{n}^{\circ} 53$; Paris, Laon, pp 37 - 69.

Charreau C. et R. Nicou. 1971. Amélioration du profil cultural dans les sols sableux et sabloargileux de la zone tropicale sèche ouestafricaine et ses incidences agronomiques. Agron. Trop. 26 : 209 - 255

Coquet Y., Vachier P. and C. Labat. 2005. Vertical variation of near-saturated hydraulic conductivity in three soil profiles. Geoderma $126: 181$ - 191.

Devineau, J. L., Fournier A. et B. Kaloga. 1997. Les sols et la végétation de Bondoukui (Ouest burkinabé) : Présentation générale et cartographie préliminaire par télédétection satellitaire (SPOT). ORSTOM éditions, Paris, $111 \mathrm{p}$.

Duval M., Angers D. A. et M. R. Laverdière. 1993. Revue de quelques facteurs régissant l'état et la stabilité de la structure du sol. Agrosol VI, 2 : 44 - 51.

FAO. 1998. World reference base for soil resources. World Soil Resources Reports, Rome, 84 : $109 \mathrm{p}$.

Fiès J. C. 1984. Analyse de la répartition du volume des pores dans les assemblages argilesquelette : Comparaison entre un modèle d'espace poral textural et les données fournies par la porosimétrie au mercure. Agronomy 4 : 891 - 899.

Hangen E., Buczko U., Bens O., Brunotte J. and R. F. Hüttl. 2002. Infiltration patterns into two soils under conventional and conservation tillage: influence of the spatial distribution of plant root structures et soil animal activity. Soil \&Tillage Research 63 : 181 - 186.

Hénin S., Gras R. et G. Monnier. 1969. Le profil cultural : L'état physique du sol et ses conséquences agronomiques. Ed. Masson, Paris, 332 p.

Jurin J. 1718. An account of some experiments shown before the Royal Society; with an enquiry into the cause of the ascent and suspension of water in capillary tubes, Philosophical Transactions of the Royal Society, $30: 739$ - 747 .

Kherabi D. et G. Monnier. 1968. Etude expérimentale de l'influence de la composition granulométrique des terres sur leur stabilité structurale. Ann. Agron. 9 (2) : $129-152$.

Martinez L. J. and J. A. Zinck. 2004. Temporal variation of soil compaction and deterioration of soil quality in pasture areas of Colombian Amazonia. Soil \&Tillage Research (75) : 3 - 17.

Nicou R. 1974. Contribution à l'amélioration de la porosité des sols tropicaux sablo-argileux de la zone tropicale sèche. Agron. Trop. 29 : $1101-1126$.

Nicou R., Ouattara B. et L. Somé. 1990. Effets des techniques d'économie de l'eau à la parcelle sur les cultures céréalières (Sorgho, Maïs, Mil) au Burkina Faso. Agron. Trop. (45) : $43-57$.

Nye P. H. and D. J. Greenland. 1960. The soil under shifting cultivation. England, Commonwealth Agricultural Bureau, 51, Farnham Royal Bucks, 156 p.

Ouattara B., Sédogo M. P. et F. Lompo. 1994. Effets de quatre types de substrats organiques sur le système poral d'un sol ferrugineux tropical sous culture de sorgho. Sci. et Techn. $21: 60$ - 77.

Ouattara B., Sédogo M. P., Assa A., Lompo F., Ouattara K. et M. Fortier. 1998. Modifications de la porosité du sol après trente-trois années de labour d'enfouissement de fumier au Burkina Faso. Cah. Agric. 7 : $9-14$.

Ouattara K., Ouattara B., Assa A. and P. M. Sédogo. 2006. Lon-term effect of ploughing and organic matter input on soil moisture characteristics of Ferric Lixisol in Burkina Faso. Soil and Tillage Research 88 : $217-224$.

Ouattara B., Ouattara K., Serpantié G., Mando A., Sedogo M. P. and A. Bationo. 2006. Intensity cultivation induced-effects on soil organic carbon dynamic in the Western Cotton area of Burkina Faso. Nutrient Cycling in Agroecosystem $76: 331$ - 339 . 
Pellerin F. M. 1980. La porosimétrie au mercure appliquée à l'étude géotechnique des sols et des roches. Bull. Liaison, Laboratoire des Ponts et Chaussée $106: 105-116$.

Ruthenberg H. 1971. Farming systems in the tropics. Clarendon Press, Oxford, $424 \mathrm{p}$.

Sarr P. L. 1981. Analyse des effets induits par l'intensification des cultures sur quelques propriétés physico-chimiques d'un sol ferrugineux tropical du Sénégal (Nioro du Rip). Thèse de 3e Cycle, USTL/ENSA, Montpellier, $100 \mathrm{p}$.

Serpantié G. et B. Ouattara. 2001. Fertilité et jachères en Afrique de l'Ouest. In : Floret $\mathrm{C}$. and R. Pontanier (Eds.). La jachère en Afrique Tropicale : De la jachère naturelle à la jachère améliorée - le point des connaissances.' Dakar, Sénégal,. John Libbey, Eurotext :, pp. 21 - 83.

Spaccini R., Zena A., Igwé C. A., Mbagwu J. S. C. and A. Piccolo. 2001. Carbohydrates in water-stable aggregates and particle size fractions of forested and cultivated soils in two contrasting tropical ecosystems. Biogeochemistry $53: 1-22$

Stengel P. 1979. Utilisation de l'analyse des systèmes de porosité pour la caractérisation de l'état physique du sol, in situ. Ann. Agron. $30: 27$ - 51.

Tisdall J. M. and J.M. Oades. 1982. Organic matter and water-stable aggregates in soils. Journal of Soil Science $33: 141$ - 163.

Vauclin M. et J. L. Chopart. 1992. L'infiltrométrie multidisque pour la détermination, in situ, des caractéristiques de la surface d'un sol gravillonnaire de Côte d'Ivoire. Agron. Trop. $46: 3$ - 11.

Wahl N. A., Bens O. Buczko U., Hangen E. and R. F. Hüttl. 2004. Effects of conventional and conservation tillage on soil hydraulic properties of a silty-loamy soil. Physical and Chemistry for the Earth $29: 821-829$. 